\title{
Editorial: Eight Journals Introduce Letters
}

At the beginning of this year the Physical Review journals introduced the article type "Letter" for eight journals: Physical Review A, Physical Review B, Physical Review C, Physical Review D, Physical Review E, Physical Review Fluids, Physical Review Materials, and Physical Review Research. At the same time, we ceased offering the article type "Rapid Communication" in these journals. Rapid Communications were first introduced in 1981 as a way to quickly publish important results that are of specialized interest to the readers of a particular Physical Review journal [1]. Since then we have published more than 40,000 Rapid Communications.

Based on broad community feedback we now publish short research articles intended for accelerated publication of important new results as Letters. Letters are an excellent way to convey significant new findings clearly and concisely to the specific readership of each journal, just as Physical Review Letters does for the physics community as a whole. With this change, we aim to maximize value and recognition for our authors by ensuring that researchers as well as funding agencies clearly understand the impact and significance of the Letters published in the Physical Review journals.

Letters will receive priority handling for prompt publication of significant new results, and this change will also clarify and streamline the transfer process of manuscripts across our journals. Authors are encouraged to follow a Letter with a more complete account as a Regular Article. The journals also accept joint submissions of a Letter and a Regular Article in accordance with the practice established for Physical Review Letters [2].

More than 150 Letters have already been published since the start of 2021, and we look forward to receiving many more Letter submissions across the journals.

Published 9 March 2021

Michael Thoennessen

Editor in Chief

American Physical Society

DOI: 10.1103/PhysRevD.103.060001

[1] David Lazarus, Changes in The Physical Review and Physical Review Letters, Phys. Rev. Lett. 45, 1605 (1980).
[2] Pierre Meystre, Editorial: Supplemental Material or Joint Submission? Phys. Rev. Lett. 118, 240001 (2017). 